\title{
The Asymptotic Diffusion Limit of a Linear Discontinuous Discretization of a Two-Dimensional Linear Transport Equation
}

\author{
CHRISTOPH BÖRGERS \\ Department of Mathematics, University of Michigan, Ann Arbor, Michigan 48109 \\ EDWARD W. LARSEN \\ Department of Nuclear Engineering, University of Michigan, Ann Arbor, Michigan 48109 \\ AND \\ MaRvin L. Adams \\ Lawrence Livermore National Laboratory, University of California, Livermore, California 94550
}

Received May 10, 1990; revised December 3, 1990

\begin{abstract}
Consider a linear transport problem, and let the mean free path and the absorption cross section be of size $\epsilon$. It is well known that one obtains a diffusion problem as $\epsilon$ tends to zero. We discretize the transport problem on a fixed mesh, independent of $\epsilon$, consider again the limit $\epsilon \rightarrow 0$, and ask whether one obtains an accurate discretization of the continuous diffusion problem. The answer is known to be affirmative for the linear discontinuous Galerkin finite element discretization in one space dimension. In this paper, we ask whether the same result holds in two space dimensions. We consider a linear discontinuous discretization based on rectangular meshes. Our main result is that the asymptotic limit of this discrete problem is not a discretization of the asymptotic limit of the continuous problem and thus that the discretization will be inaccurate in the asymptotic regime under consideration. We also propose a modified scheme which has the correct asymptotic behavior for spatially periodic problems, although not always for problems with boundaries. We present numerical results confirming our formal asymptotic analysis. (c) 1992 Academic Press, Inc.
\end{abstract}

\section{INTRODUCTION}

Linear transport equations describe processes in which particles (typically neutrons, electrons, or photons) undergo linear interactions with a dense host of background nuclei, but do not interact with each other. Such equations find application in nuclear reactor analysis, the conduction of electrons in solids, and the propagation of photons in planetary and stellar atmospheres. We refer to [1] for the theory of linear transport, and to [2] for numerical techniques for such problems.

Numerical transport solutions are often costly to obtain, and various approximations to transport theory, par- ticularly diffusion theory, are frequently used instead. The relationship between transport and diffusion theory has, in the past two decades, become clarified by the use of an asymptotic analysis $[3,4]$. Namely, it has been shown that diffusion theory is an asymptotic limit of transport theory for problems in which the physical domain is optically thick, i.e., many mean free paths in diameter, and the probable number of particles exiting a collision is nearly equal to unity. In a recent series of papers $[5,6]$ the following question has been considered: If one applies the same asymptotic expansion to a discretized transport problem on a fixed mesh, will one obtain an accurate discretization of the diffusion problem? If the answer is yes, then one may be able to obtain accurate results using optically thick mesh cells. This is an important issue for thermal photon transport problems, in which the meshes used in practice tend to have optically thick cells. There are related, but more complicated, issues with electron transport.

In [6], it was shown theoretically and numerically that the linear discontinuous (LD) finite element method in one space dimension is highly accurate in the asymptotic regime discussed above. The LD method has in fact found wide use in transport applications for one-dimensional problems [7], two-dimensional problems on rectangular meshes [8-10], and two-dimensional problems on triangular meshes [11]. The theoretical and experimental success of the LD method for asymptotically diffusive one-dimensional problems leads one to ask whether a similar result holds for two-dimensional problems. The purpose of the present paper is to show that for two-dimensional $(x, y)$ - 
geometry, on a rectangular spatial mesh, the LD scheme does not, except for essentially one-dimensional problems, asymptotically limit to a consistent discretization of the continuous diffusion problem. Our result leaves open the possibility that the LD scheme does have the correct limit when applied on a triangular mesh, or that a higher-order finite element method has the correct limit for a rectangular mesh.

We specifically consider two-dimensional transport problems of the following form. Let $Q=Q(\mathbf{x})$ be a given function of $\mathbf{x}=(x, y) \in \mathbb{R}^{2}$ with period 1 in both coordinate directions:

$$
Q(x+1, y) \equiv Q(x, y+1) \equiv Q(x, y) .
$$

(The variables $\mathbf{x}$ are the spatial variables, and $Q$ is a prescribed interior source of particles.) Let $D$ denote the disk in the plane, centered at the origin, with radius one. We shall use the notation $\boldsymbol{\Omega}=(\mu, \eta)$ for points in $D$. (The variables $\boldsymbol{\Omega}$ are the "angular" variables, denoting the direction of particle motion.) The unknown is a function

$$
\psi=\psi(\mathbf{x}, \mathbf{\Omega}), \quad \mathbf{x} \in \mathbb{R}^{2}, \mathbf{\Omega} \in D,
$$

which defines the particle flux at $(\mathbf{x}, \mathbf{\Omega})$. One can also think of $\psi$ as a function defined on the cross product of $\mathbb{R}^{2}$ with the unit sphere $S^{2} \subseteq R^{3}$, by setting

$$
\psi\left(\mathbf{x}, \mu, \eta, \pm \sqrt{1-\mu^{2}-\eta^{2}}\right):=\psi(\mathbf{x}, \mu, \eta) .
$$

The transport problem is

$$
\begin{gathered}
\mathbf{\Omega} \cdot \boldsymbol{\nabla} \psi(\mathbf{x}, \mathbf{\Omega})+\sigma_{T}(\mathbf{x}) \psi(\mathbf{x}, \mathbf{\Omega}) \\
=\frac{\sigma_{T}(\mathbf{x})-\sigma_{A}(\mathbf{x})}{4 \pi} \int_{S^{2}} \psi\left(\mathbf{x}, \mathbf{\Omega}^{\prime}\right) d \mathbf{\Omega}^{\prime} \\
\quad+Q(\mathbf{x}), \mathbf{x} \in \mathbb{R}^{2}, \mathbf{\Omega} \in D, \\
\psi(x+1, y, \mathbf{\Omega}) \\
\equiv \psi(x, y+1, \mathbf{\Omega}) \equiv \psi(x, y, \mathbf{\Omega}) \\
\quad \text { for all }(x, y) \text { and } \mathbf{\Omega},
\end{gathered}
$$

where $\sigma_{T}$ and $\sigma_{A}$ are given functions, the total and absorption cross sections, with $0 \leqslant \sigma_{A}(\mathbf{x}) \leqslant \sigma_{T}(\mathbf{x}) . \sigma_{T}$ and $\sigma_{A}$ are assumed to be periodic in both coordinate directions with period 1.

We shall restrict ourselves to periodic problems. However, much of the analysis of the following sections applies to problems with boundaries. Issues related to boundary conditions will briefly be discussed in Section 7, and more fully in a future publication.

We describe the physical situation modeled by Eq. (1). Consider particles with constant speed $v$ moving among fixed nuclei. The flux of particles at the location $\mathbf{x}$ with direction vector $\Omega$ is $\psi(x, \Omega)=v n(x, \Omega)$, where $n(x, \Omega)$ is the particle density. The particles may collide with nuclei, and in so doing are either absorbed or scattered. When a particle is scattered, it exits the collision with a random direction vector $\Omega \in S^{2}$, and the probability distribution for this direction vector is uniform. The number of collisions per unit time for a particle (the collision rate) is given by $v \sigma_{T}$, and the number of collisions resulting in absorption per unit time (the absorption rate) is given by $v \sigma_{A}$. Also, the mean distance between collisions (the mean free path) is $\sigma_{T}^{-1}$, and the mean distance between absorptions is $\sigma_{A}^{-1}$. It is assumed that $\sigma_{T}$ and $\sigma_{A}$ do not depend on $\boldsymbol{\Omega}$. In addition, there is an interior particle source, with the periodic density function $Q(\mathbf{x})$. In steady state, the flux satisfies Eqs. (1) and (2).

To angularly discretize Eqs. (1) and (2), we make the discrete ordinates approximation [2]. Thus, we replace the integral in Eq. (1) by a sum, using a numerical scheme for evaluating integrals of scalar functions over the unit sphere. We then obtain a system of equations of the form

$$
\begin{aligned}
\boldsymbol{\Omega}_{m} & \cdot \nabla \psi_{m}(\mathbf{x})+\sigma_{T}(\mathbf{x}) \psi_{m}(\mathbf{x}) \\
& =\left[\sigma_{T}(\mathbf{x})-\sigma_{A}(\mathbf{x})\right] \sum_{k=1}^{N} w_{k} \psi_{k}(\mathbf{x})+Q(\mathbf{x}),
\end{aligned}
$$

$m=1, \ldots, N$, with the periodicity conditions

$$
\psi_{m}(x+1, y) \equiv \psi_{m}(x, y+1) \equiv \psi_{m}(x, y) .
$$

Note that the weights $w_{k}$ are scaled in such a way that the denominator $4 \pi$ in Eq. (1) is not present in Eq. (3).

We shall not be concerned with the choice of the angular quadrature scheme, i.e., with the specific choice of the $\boldsymbol{\Omega}_{k}$ and the $w_{k}$. We refer to [2, Section 4.2], for a discussion of standard angular quadrature schemes. However, we shall make the following requirements, which are in fact satisfied by standard quadrature sets used in two-dimensional transport codes. First, we require the quadrature scheme to exactly integrate polynomials in $\mu$ and $\eta$ of degree less than or equal to 2 . Thus,

$$
\begin{aligned}
\sum_{k=1}^{N} w_{k} & =1, \\
\sum_{k=1}^{N} w_{k} \mu_{k} & =\sum_{k=1}^{N} w_{k} \eta_{k}=0, \\
\sum_{k=1}^{N} w_{k} \mu_{k}^{2} & =\sum_{k=1}^{N} w_{k} \eta_{k}^{2}=\frac{1}{3}, \\
\sum_{k=1}^{N} w_{k} \mu_{k} \eta_{k} & =0 .
\end{aligned}
$$


Second, we require both components of $\boldsymbol{\Omega}_{m}$ to be nonzero for all $m$ :

$$
\mu_{m} \neq 0, \quad \eta_{m} \neq 0, \quad m=1, \ldots, N
$$

Third, we require the quadrature scheme to be symmetric, in the sense that

$$
\begin{aligned}
\left\{\mu_{1}, \ldots, \mu_{N}\right\} & =\left\{-\mu_{1}, \ldots,-\mu_{N}\right\} \\
& =\left\{\eta_{1}, \ldots, \eta_{N}\right\}=\left\{-\eta_{1}, \ldots,-\eta_{N}\right\}
\end{aligned}
$$

and

$$
\left(\left|\mu_{m}\right|,\left|\eta_{m}\right|\right)=\left(\left|\mu_{n}\right|,\left|\eta_{n}\right|\right) \Rightarrow w_{m}=w_{n} .
$$

In a certain asymptotic limit, characterized by large $\sigma_{T}$ and small $\sigma_{A}$, Eq. (3) reduces to a diffusion equation. This so-called asymptotic diffusion limit is well known $[3,4]$. For the convenience of the reader, we shall review it in Section 2.

We shall study the linear discontinuous Galerkin finite element method on rectangular meshes for Eqs. (3) and (4). For an introduction to such finite element methods, see, for example, [12]. Thus the $\psi_{m}$ are approximated by functions that are piecewise linear, i.e., linear within each cell of the mesh, but possibly discontinuous at the cell edges. The linear discontinuous scheme is described in detail in Section 3. One might think that on a rectangular mesh, piecewise bilinear approximations would be more natural than piecewise linear ones. But the cost per mesh cell is lower for piecewise linear approximations; these approximations have been successfully utilized in neutron transport applications [8-10], and we are not aware of any previously known convincing objection against the piecewise linear scheme. However, our results will show that the scheme does indeed have a serious defect.

Recently, asymptotic diffusion limits have also been derived for one-dimensional spatial discretizations of transport problems $[5,6]$. In Section 4, we present such an analysis for the two-dimensional linear discontinuous Galerkin method. Our main result is that the diffusion limit of the discrete scheme is in general inconsistent with that of the continuous problem. The discretization therefore will be inaccurate for large $\sigma_{T}$ and small $\sigma_{A}$. This result does not contradict the favorable conclusions described in [8-10] for the linear discontinuous method on a rectangular mesh applied to neutron transport problems. This is because in neutron transport applications, the spatial cells are typically not more than a few mean free paths thick. Our analysis is more relevant to applications in electron and photon transport problems.

In Section 5, we show that a modification of the scheme, suggested by the asymptotic analysis, leads to correct asymptotic behavior for the spatially periodic problems considered here. However, even this modification does not appear to lead to the right behavior in the diffusion limit for certain problems with nonperiodic boundaries.

Numerical results illustrating our theory are given in Section 6. We believe that much of the strategy of the calculations of Sections 4 and 5 can be applied to other discretizations of transport equations. We discuss possible extensions and applications of our work in Section 7.

\section{THE ASYMPTOTIC DIFFUSION LIMIT OF THE TWO-DIMENSIONAL DISCRETE ORDINATES EQUATIONS}

Let us consider the following scaling of Eq. (3):

$$
\mathbf{\Omega}_{m} \cdot \nabla \psi_{m}+\frac{\sigma_{T}}{\varepsilon} \psi_{m}=\left(\frac{\sigma_{T}}{\varepsilon}-\varepsilon \sigma_{A}\right) \sum_{k=1}^{N} w_{k} \psi_{k}+\varepsilon Q
$$

for $\varepsilon>0$. We are interested in the limit $\varepsilon \rightarrow 0$. The scaling of $\sigma_{T}$ expresses that we wish to consider small mean free paths. The motivation of the scaling of $\sigma_{A}$ will become clear from the asymptotic analysis presented below: If $\sigma_{A}<O(\varepsilon)$, absorption has no effect at all asymptotically. If $\sigma_{A}>O(\varepsilon)$, absorption dominates asymptotically. The source $Q$ is scaled in such a way that the solution remains of size $O(1)$ as $\varepsilon \rightarrow 0$.

We now consider an asymptotic expansion

$$
\psi_{m}=\psi_{m}^{(0)}+\varepsilon \psi_{m}^{(1)}+\varepsilon^{2} \psi_{m}^{(2)}+\cdots
$$

We shall call quantities with a superscript $(n) n$ th-order quantities. Inserting the asymptotic expansion into Eq. (12) and equating the coefficients of $\varepsilon^{-1}$, we find

$$
\psi_{m}^{(0)}(\mathbf{x})=\sum_{k=1}^{N} w_{k} \psi_{k}^{(0)}(\mathbf{x})=: \Psi^{(0)}(\mathbf{x})
$$

for all $m$. We call Eq. (13) the zeroth-order equation. Equating the coefficients of $\varepsilon^{0}$, we obtain the first-order equation:

$$
\boldsymbol{\Omega}_{m} \cdot \nabla \psi_{m}^{(0)}+\sigma_{T} \psi_{m}^{(1)}=\sigma_{T} \sum_{k=1}^{N} w_{k} \psi_{k}^{(1)}
$$

Also, equating the coefficients of $\varepsilon^{1}$, we obtain the secondorder equation:

$$
\begin{aligned}
\boldsymbol{\Omega}_{m} & \cdot \nabla \psi_{m}^{(1)}+\sigma_{T} \psi_{m}^{(2)} \\
& =\sigma_{T} \sum_{k=1}^{N} w_{k} \psi_{k}^{(2)}-\sigma_{A} \Psi^{(0)}+Q
\end{aligned}
$$


Let us define

$$
\Psi^{(1)}:=\sum_{k=1}^{N} w_{k} \psi_{k}^{(1)}
$$

Then Eq. (14) can be written as

$$
\psi_{m}^{(1)}=\Psi^{(1)}-\frac{1}{\sigma_{T}} \mathbf{\Omega}_{m} \cdot \nabla \Psi^{(0)}
$$

We arrange Eq. (15) as

$$
\sigma_{T}\left[\psi_{m}^{(2)}-\sum_{k=1}^{N} w_{k} \psi_{k}^{(2)}\right]=-\boldsymbol{\Omega}_{m} \cdot \nabla \psi_{m}^{(1)}-\sigma_{A} \Psi^{(0)}+Q
$$

Multiplying by $w_{m}$, summing over $m$, and using Eq. (5), we obtain

$$
\sum_{k-1}^{N} w_{k} \boldsymbol{\Omega}_{k} \cdot \nabla \psi_{k}^{(1)}+\sigma_{A} \Psi^{(0)}=Q
$$

Inserting Eq. (16) into Eq. (17), and using Eq. (6), we obtain

$$
-\sum_{k=1}^{N} w_{k} \boldsymbol{\Omega}_{k} \cdot \nabla \frac{1}{\sigma_{T}} \boldsymbol{\Omega}_{k} \cdot \nabla \Psi^{(0)}+\sigma_{A} \Psi^{(0)}=Q .
$$

Finally, using Eqs. (7) and (8), we obtain the diffusion equation

$$
-\nabla \cdot \frac{1}{3 \sigma_{T}(\mathbf{x})} \nabla \Psi^{(0)}(\mathbf{x})+\sigma_{A}(\mathbf{x}) \Psi^{(0)}(\mathbf{x})=Q(\mathbf{x}) .
$$

The calculations of Sections 4 and 5 will be discrete variants of the one described above. To clarify the strategy, we summarize the essential steps.

The zeroth-order equations express that zeroth-order quantities are isotropic, i.e., independent of $m$. The firstorder equations express anisotropic first-order quantities in terms of zeroth-order quantities and isotropic first-order quantities. The second-order equations involve secondorder quantities, but these quantities do not appear in the solvability conditions obtained by averaging over the angular variable. Anisotropic first-order quantities are eliminated from thcse solvability conditions using the firstorder equations. The remaining isotropic first-order quantities drop out because of the mild accuracy assumptions on the angular quadrature scheme listed in Section 1. The solvability conditions for the second-order equations then result in a diffusion equation for the zeroth order quantities.

\section{THE LINEAR DISCONTINUOUS GALERKIN FINITE ELEMENT METHOD}

In this section, we present a derivation of the linear discontinuous Galerkin finite element method for Eqs. (3) and (4). Consider first a single advection equation of the form

$$
(\boldsymbol{\Omega} \cdot \boldsymbol{\nabla}) \psi(\mathbf{x})+\sigma_{T}(\mathbf{x}) \psi(\mathbf{x})=Q(\mathbf{x})
$$

where $\Omega$ is a non-zero vector in the plane. As before, $Q$ is assumed to be periodic with period 1 in both coordinate directions, and we seek a periodic solution $\psi$. We first present the linear discontinuous Galerkin finite element method for Eq. (20). The generalization to Eqs. (3) and (4) will then be straightforward.

Consider a partition of $(0,1)^{2}$ into finitely many polygonal cells. Via periodic extension, we think of it as a partition of $\mathbb{R}^{2}$ into polygonal cells. Let $K$ be one of the cells. Let $\psi$ be a solution of Eq. (20), and let $\phi$ be an arbitrary smooth function on $K$. Then we have

$$
\begin{gathered}
\int_{K}\left[(\boldsymbol{\Omega} \cdot \nabla) \psi(\mathbf{x})+\sigma_{T}(\mathbf{x}) \psi(\mathbf{x})\right. \\
-Q(\mathbf{x})] \phi(\mathbf{x}) d \mathbf{x}=0 .
\end{gathered}
$$

Equation (21) is a weak formulation of Eq. (20), and one can attempt to obtain a discretization scheme from it by restricting $\psi$ and $\phi$ to a space of piecewise polynomials. For instance, one might seek an approximation $\psi^{h}$ for $\psi$ that is periodic, piecewise linear, i.e., linear on each $K$, but possibly discontinuous, and such that Eq. (21) holds for every linear $\phi=\phi^{h}$ on $K$. Clearly such an approach does not immediately lead to a reasonable scheme, because the values of $\psi^{h}$ on different cells $K$ are unrelated to each other. However, consider the following reformulation of Eq. (21), obtained by integration by parts:

$$
\begin{aligned}
\int_{K}\{ & -(\boldsymbol{\Omega} \cdot \boldsymbol{\nabla}) \phi(\mathbf{x}) \psi(\mathbf{x}) \\
& \left.+\left\lceil\sigma_{T}(\mathbf{x}) \psi(\mathbf{x})-Q(\mathbf{x})\right\rceil \phi(\mathbf{x})\right\} d \mathbf{x} \\
& +\int_{\partial K} \psi(\mathbf{x}) \phi(\mathbf{x})\left(\mathbf{\Omega} \cdot \mathbf{n}_{K}(\mathbf{x})\right) d s(\mathbf{x})=0 .
\end{aligned}
$$

Here $\mathbf{n}_{K}(\mathbf{x})$ denotes the exterior unit normal vector on $\partial K$. Let us again consider replacing $\psi$ by a piecewise linear, possibly discontinuous $\psi^{h}$. We make the preliminary convention that for $\mathbf{x} \in \partial K, \psi^{h}(\mathbf{x})$ denotes the interior value of $\psi^{h}$ in $\mathbf{x}$, i.e., the limit of $\psi^{h}(\mathbf{y})$ as $\mathbf{y}$ tends to $\mathbf{x}$ from inside $K$. 
The scheme that we have previously rejected because it fails to couple different cells to each other can then be stated as

$$
\begin{aligned}
\int_{K}\{ & -(\mathbf{\Omega} \cdot \nabla) \phi^{h}(\mathbf{x}) \psi^{h}(\mathbf{x}) \\
& \left.+\left[\sigma_{T}(\mathbf{x}) \psi^{h}(\mathbf{x})-Q(\mathbf{x})\right] \phi^{h}(\mathbf{x})\right\} d \mathbf{x} \\
& +\int_{\partial K} \psi^{h}(\mathbf{x}) \phi^{h}(\mathbf{x})\left(\mathbf{\Omega} \cdot \mathbf{n}_{K}(\mathbf{x})\right) d s(\mathbf{x})=0
\end{aligned}
$$

for all linear functions $\phi^{h}$ on $K$. The discontinuous Galerkin finite element method [12] is obtained when the convention is changed such that $\psi^{h}(\mathbf{x}), \mathbf{x} \in \partial K$, denotes the upstream value rather than the interior value. To state this more explicitly, let us define

$$
\partial^{-} K:=\left\{\mathbf{x} \in \partial K: \mathbf{\Omega} \cdot \mathbf{n}_{K}(\mathbf{x})<0\right\} .
$$

Then the new convention is that $\psi^{h}(\mathbf{x})$ denotes the exterior value on $\partial^{-} K$, and the interior value on $\partial K-\partial^{-} K$. For $\mathbf{x} \in \partial^{-} K$, we define $\psi^{h+}(\mathbf{x})$ to be the downstream, i.e., the interior value of $\psi^{h}$ in $\mathbf{x}$, and $\psi^{h-}(\mathbf{x})$ to be the upstream, i.e., the exterior value. Then the scheme can be stated as

$$
\begin{aligned}
& \int_{K}\left[(\boldsymbol{\Omega} \cdot \nabla) \psi^{h}(\mathbf{x})+\sigma_{T}(\mathbf{x}) \psi^{h}(\mathbf{x})-Q(\mathbf{x})\right] \\
& \quad \times \phi^{h}(\mathbf{x}) d \mathbf{x}-\int_{\hat{\sigma}^{-} K}\left[\psi^{h+}(\mathbf{x})-\psi^{h-}(\mathbf{x})\right] \\
& \quad \times \phi^{h}(\mathbf{x})\left(\mathbf{\Omega} \cdot \mathbf{n}_{K}(\mathbf{x})\right) d s(\mathbf{x})=0 .
\end{aligned}
$$

Up to notation, this equation is identical with Eq. (3.11) of [12]. It is required to hold for all linear $\phi^{h}$ on $K$.

This concludes our discussion of the method for the advection problem (20). The scheme can be directly applied to Eqs. (3) and (4) by regarding the entire right-hand side of Eq. (3) as a source term.

We now consider the special case when the partition of $(0,1)^{2}$ into polygons is given by a rectangular mesh, and take $\psi^{h}$ to be piecewise linear, but possibly discontinuous, and the test functions $\phi^{h}$ to be lincar. To simplify the notation, we restrict ourselves to the case of constant cross sections $\sigma_{T}$ and $\sigma_{A}$. The cell vertices are denoted by

$$
\left(x_{i \pm 1 / 2}, y_{j \pm 1 / 2}\right), \quad 1 \leqslant i \leqslant I, 1 \leqslant j \leqslant J,
$$

with

$$
0=x_{1 / 2}<x_{3 / 2}<\cdots<x_{I-1 / 2}<x_{I+1 / 2}=1
$$

and

$$
0=y_{1 / 2}<y_{3 / 2}<\cdots<y_{J-1 / 2}<y_{J+1 / 2}=1 \text {. }
$$

We introduce the notation:

$$
\begin{aligned}
& h_{i}:=x_{i+1 / 2}-x_{i-1 / 2}, \\
& k_{j}:=y_{j+1 / 2}-y_{j-1 / 2}, \\
& x_{i}:=\frac{1}{2}\left(x_{i-1 / 2}+x_{i+1 / 2}\right), \\
& y_{j}:=\frac{1}{2}\left(y_{j-1 / 2}+y_{j+1 / 2}\right) .
\end{aligned}
$$

We drop the superscript $h$, and denote by $\psi_{m}$ the approximation to the solution of Eqs. (3) and (4). In the $(i, j)$ th cell, i.e., in

$$
\left(x_{i-1 / 2}, x_{i+1 / 2}\right) \times\left(y_{j-1 / 2}, y_{j+1 / 2}\right),
$$

$\psi_{m}$ is linear and therefore of the form

$$
\begin{gathered}
\psi_{m}(x, y)=\psi_{m, i, j}+\frac{2}{h_{i}}\left(x-x_{i}\right) \xi_{m, i, j} \\
+\frac{2}{k_{j}}\left(y-y_{j}\right) \chi_{m, i, j} .
\end{gathered}
$$

Subscripts of the dependent variables are always understood to be evaluated modulo $I$ or modulo $J$, i.e., $\psi_{m, i+I, j}=\psi_{m, i, j+J}=\psi_{m, i, j}$, etc. The downstream edge values of $\psi_{m}$ are of the form

$$
\psi_{m, i, j \pm 1 / 2}+\frac{2}{h_{i}}\left(x-x_{i}\right) \xi_{m, i, j \pm 1 / 2}
$$

and

$$
\psi_{m, i \pm 1 / 2, j}+\frac{2}{k_{j}}\left(y-y_{j}\right) \chi_{m, i \pm 1 / 2, j}
$$

Comparing Eqs. (27) and (28) with Eq. (26), we find relations between the cell variables and the edge variables. To express these relations in a compact form, we introduce the notation

$$
\alpha_{m}:=\frac{\mu_{m}}{\left|\mu_{m}\right|}, \quad \beta_{m}:=\frac{\eta_{m}}{\left|\eta_{m}\right|} .
$$

Then we have

$$
\begin{aligned}
\psi_{m, i+1 / 2, j}= & \frac{1+\alpha_{m}}{2}\left(\psi_{m, i, j}+\xi_{m, i, j}\right) \\
& +\frac{1-\alpha_{m}}{2}\left(\psi_{m, i+1, j}-\xi_{m, i+1, j}\right), \\
\psi_{m, i, j+1 / 2}= & \frac{1+\beta_{m}}{2}\left(\psi_{m, i, j}+\chi_{m, i, j}\right) \\
& +\frac{1-\beta_{m}}{2}\left(\psi_{m, i, j+1}-\chi_{m, i, j+1}\right),
\end{aligned}
$$




$$
\begin{aligned}
& \chi_{m, i+1 / 2, j}=\frac{1+\alpha_{m}}{2} \chi_{m, i, j}+\frac{1-\alpha_{m}}{2} \chi_{m, i+1, j}, \\
& \xi_{m, i, j+1 / 2}=\frac{1+\beta_{m}}{2} \xi_{m, i, j}+\frac{1-\beta_{m}}{2} \xi_{m, i+1, j} .
\end{aligned}
$$

We also define the spatial moments of the source:

$$
\begin{aligned}
Q_{i, j} & :=\frac{1}{h_{i} k_{j}} \int_{x_{i-1 / 2}}^{x_{i+1 / 2}} \int_{y_{j-1 / 2}}^{y_{j+1 / 2}} Q(x, y) d y d x, \\
Q_{x, i, j} & :=\frac{6}{h_{i}^{2} k_{j}} \int_{x_{i-1 / 2}}^{x_{i+1 / 2}} \int_{y_{j-1 / 2}}^{y_{j+1 / 2}}\left(x-x_{i}\right) Q(x, y) d y d x, \\
Q_{y, i, j} & :=\frac{6}{h_{i} k_{j}^{2}} \int_{x_{i-1 / 2}}^{x_{i+1 / 2}} \int_{y_{j-1 / 2}}^{y_{j+1 / 2}}\left(y-y_{j}\right) Q(x, y) d y d x .
\end{aligned}
$$

Note that if $Q$ is linear on the $(i, j)$ th cell, then

$$
\begin{aligned}
Q(x, y)= & Q_{i, j}+\frac{2}{h_{i}}\left(x-x_{i}\right) Q_{x, i, j} \\
& +\frac{2}{k_{j}}\left(y-y_{j}\right) Q_{y, i, j} .
\end{aligned}
$$

Equation (25) with $\phi(x, y)=1$ now translates into

$$
\begin{aligned}
& \frac{\mu_{m}}{h_{i}}\left(\psi_{m, i+1 / 2, j}-\psi_{m, i-1 / 2, j}\right) \\
& \quad+\frac{\eta_{m}}{k_{j}}\left(\psi_{m, i, j+1 / 2}-\psi_{m, i, j-1 / 2}\right)+\sigma_{T} \psi_{m, i, j} \\
& =\left(\sigma_{T}-\sigma_{A}\right) \sum_{k=1}^{N} w_{k} \psi_{k, i, j}+Q_{i, j} .
\end{aligned}
$$

Similarly, $\phi(x, y)=x-x_{i}$ gives

$$
\begin{aligned}
& \frac{3 \mu_{m}}{h_{i}}\left(\psi_{m, i+1 / 2, j}+\psi_{m, i-1 / 2, j}-2 \psi_{m, i, j}\right) \\
& \quad+\frac{\eta_{m}}{k_{j}}\left(\xi_{m, i, j+1 / 2}-\xi_{m, i, j-1 / 2}\right)+\sigma_{T} \xi_{m, i, j} \\
& =\left(\sigma_{T}-\sigma_{A}\right) \sum_{k=1}^{N} w_{k} \xi_{k, i, j}+Q_{x, i, j}
\end{aligned}
$$

and $\phi(x, y)=y-y_{j}$ gives

$$
\begin{aligned}
\frac{3 \eta_{m}}{k_{j}}\left(\psi_{m, i, j+1 / 2}+\psi_{m, i, j-1 / 2}-2 \psi_{m, i, j}\right) & \\
& +\frac{\mu_{m}}{h_{i}}\left(\chi_{m, i+1 / 2, j}-\chi_{m, i-1 / 2, j}\right)+\sigma_{T} \chi_{m, i, j} \\
= & \left(\sigma_{T}-\sigma_{A}\right) \sum_{k=1}^{N} w_{k} \chi_{k, i, j}+Q_{y, i, j} .
\end{aligned}
$$

Equations (30)-(33) and (34)-(36) describe the linear discontinuous Galerkin method on rectangular meshes.

We note that one could allow variable cross sections $\sigma_{T}$ and $\sigma_{A}$ simply by replacing $\sigma_{T}$ and $\sigma_{A}$ in Eqs. (34)-(36) by $\sigma_{T, i, j}$ and $\sigma_{A, i, j}$. For the resulting scheme, the analysis of this paper can be carried out without any essential changes.

We shall also consider the following modification of Eqs. (35) and (36); compare, for example, [6]:

$$
\begin{aligned}
& \frac{0 \mu_{m}}{h_{i}}\left(\psi_{m, i+1 / 2, j}+\psi_{m, i-1 / 2, j}-2 \psi_{m, i, j}\right) \\
& \quad+\frac{y \eta_{m}}{k_{j}}\left(\xi_{m, i, j+1 / 2}-\xi_{m, i, j-1 / 2}\right)+\sigma_{T} \xi_{m, i, j} \\
& =\left(\sigma_{T}-\sigma_{A}\right) \sum_{k=1}^{N} w_{k} \xi_{k, i, j}+Q_{x, i, j}
\end{aligned}
$$

and

$$
\begin{aligned}
\frac{\theta \eta_{m}}{k_{j}}\left(\psi_{m, i, j+1 / 2}+\psi_{m, i, j-1 / 2}-2 \psi_{m, i, j}\right) & \\
& +\frac{\gamma \mu_{m}}{h_{i}}\left(\chi_{m, i+1 / 2, j}-\chi_{m, i-1 / 2, j}\right)+\sigma_{T} \chi_{m, i, j} \\
= & \left(\sigma_{T^{-}}-\sigma_{A}\right) \sum_{k=1}^{N} w_{k} \chi_{k, i, j}+Q_{y, i, j} .
\end{aligned}
$$

Here $\theta$ and $\gamma$ are free parameters, and Eqs. (35) and (36) are obtained by setting $\theta=3$ and $\gamma=1$. We call the method defined by Eqs. (30)-(33), (34), (37), and (38) the modified linear discontinuous (MLD) method. Modifications of this kind have been used in practice [6]. The parameters $\theta$ and $\gamma$ could, of course, also be chosen as functions of $m, i, j, \sigma_{T}$, and $\sigma_{A}$.

In one space dimension, there is no parameter analogous to $\gamma$, and one can show that the replacement of $\theta=3$ by $\theta=1$ is equivalent to lumping the mass matrix if the canonical basis of the space of piecewise linear, possibly discontinuous functions is used. (The "canonical" basis consists of the piecewise linear functions that are one at one of the endpoints of one of the mesh intervals, zero at the other endpoint of that interval, and on all other intervals.) We know no similar interpretation of the parameters $\theta$ and $\gamma$ in two space dimensions.

The parameters $\theta$ and $\gamma$ have been introduced in such a way that linear solutions are preserved. More precisely, consider an infinite medium with constant cross sections and with a linearly varying source,

$$
Q(x, y)=Q+x Q_{x}+y Q_{y}
$$

where $Q, Q_{x}$, and $Q_{y}$ are constants. Then it is a matter of 
simple algebra to show that Eq. (3) has exactly one solution that is also linearly varying in $x$ and $y$ :

$$
\begin{aligned}
\psi_{m}(x, y)= & \frac{Q}{\sigma_{A}}-\frac{\mu_{m} Q_{x}+\eta_{m} Q_{y}}{\sigma_{T} \sigma_{A}} \\
& +x \frac{Q_{x}}{\sigma_{A}}+y \frac{Q_{y}}{\sigma_{A}}
\end{aligned}
$$

It is easy to show that the modified linear discontinuous scheme preserves this exact solution for arbitrary choices of the parameters $\theta$ and $\gamma$.

It is natural to require $\theta \rightarrow 3$ and $\gamma \rightarrow 1$ as the mesh widths $h_{i}$ and $k_{j}$ tend to zero, with all other data fixed; then the MLD scheme reduces to the standard LD scheme for small mesh widths. However, for fixed nonzero mesh widths, one might attempt to improve the accuracy of the scheme by using $\theta \neq 3$ and $\gamma \neq 1$. In Section 4, we shall show that the MLD scheme behaves incorrectly in the diffusion limit unless $\gamma \rightarrow 0$ as the scaling parameter $\varepsilon$ tends to zero on a fixed mesh.

\section{THE ASYMPTOTIC DIFFUSION LIMIT FOR $\theta>0$ AND $\gamma>0$}

We introduce the scaling of Section 2 into Eqs. (34), (37), and (38). Thus we consider the equations

$$
\begin{aligned}
& \frac{\mu_{m}}{h_{i}}\left(\psi_{m, i+1 / 2, j}-\psi_{m, i-1 / 2, j}\right) \\
&+\frac{\eta_{m}}{k_{j}}\left(\psi_{m, i, j+1 / 2}-\psi_{m, i, j-1 / 2}\right)+\frac{\sigma_{T}}{\varepsilon} \psi_{m, i, j} \\
&=\left(\frac{\sigma_{T}}{\varepsilon}-\varepsilon \sigma_{A}\right) \sum_{k=1}^{N} w_{k} \psi_{k, i, j}+\varepsilon Q_{i, j}, \\
& \frac{\theta \mu_{m}}{h_{i}}\left(\psi_{m, i+1 / 2, j}+\psi_{m, i-1 / 2, j}-2 \psi_{m, i, j}\right) \\
& \quad+\frac{\gamma \eta_{m}}{k_{j}}\left(\xi_{m, i, j+1 / 2}-\xi_{m, i, j-1 / 2}\right)+\frac{\sigma_{T}}{\varepsilon} \xi_{m, l, j} \\
&=\left(\frac{\sigma_{T}}{\varepsilon}-\varepsilon \sigma_{A}\right) \sum_{k=1}^{N} w_{k} \xi_{k, i, j}+\varepsilon Q_{x, i, j}, \\
& \frac{\theta \eta_{m}}{k_{j}}\left(\psi_{m, i, j+1 / 2}+\psi_{m, i, j-1 / 2}-2 \psi_{m, i, j}\right) \\
& \quad+\frac{\gamma \mu_{m}}{h_{i}}\left(\chi_{m, i+1 / 2, j}-\chi_{m, i-1 / 2}, j\right)+\frac{\sigma_{T}}{\varepsilon} \chi_{m, i, j} \\
&=\left(\frac{\sigma_{T}}{\varepsilon}-\varepsilon \sigma_{A}\right) \sum_{k=1}^{N} w_{k} \chi_{k, i, j}+\varepsilon Q_{y, i, j},
\end{aligned}
$$

together with Eqs. (30)-(33).
We use Eqs. (30)-(33) to eliminate the cell edge variables $\psi_{m, i \pm 1 / 2, j}, \psi_{m, i, j \pm 1 / 2}, \xi_{m, i, j \pm 1 / 2}, \chi_{m, i \pm 1 / 2, j}$. Using the notation defined in Eq. (29), we obtain

$$
\begin{aligned}
& \frac{\mu_{m}}{h_{i}}\left[\frac{1+\alpha_{m}}{2}\left(\psi_{m, i, j}+\xi_{m, i, j}-\psi_{m, i-1, j}-\xi_{m, i-1, j}\right)\right. \\
& \left.\quad+\frac{1-\alpha_{m}}{2}\left(\psi_{m, i+1, j}-\xi_{m, i+1, j}-\psi_{m, i, j}+\xi_{m, i, j}\right)\right] \\
& \quad \times \frac{\eta_{m}}{k_{j}}\left[\frac{1+\beta_{m}}{2}\left(\psi_{m, i, j}+\chi_{m, i, j}-\psi_{m, i, j-1}-\chi_{m, i, j-1}\right)\right. \\
& \quad+\frac{1-\beta_{m}}{2}\left(\psi_{m, i, j+1}-\chi_{m, i, j+1}\right. \\
& \left.\left.\quad-\psi_{m, i, j}+\chi_{m, i, j}\right)\right]+\frac{\sigma_{T}}{\varepsilon} \psi_{m, i, j} \\
& =\left(\frac{\sigma_{T}}{\varepsilon}-\varepsilon \sigma_{A}\right) \sum_{k} w_{k} \psi_{k, i, j}+\varepsilon Q_{i j}
\end{aligned}
$$

$$
\begin{aligned}
\frac{\theta \mu_{m}}{h_{i}}\left[\frac { 1 } { 2 } \left(\alpha_{m}\right.\right. & \left.\psi_{m, i, j}+\xi_{m, i, j}+\psi_{m, i-1, j}+\xi_{m, i-1, j}-2 \psi_{m, i, j}\right) \\
& +\frac{1-\alpha_{m}}{2}\left(\psi_{m, i+1, j}-\xi_{m, i+1, j}\right. \\
& \left.\left.+\psi_{m, i, j}-\xi_{m, i, j}-2 \psi_{m, i, j}\right)\right] \\
& \times \frac{\gamma \eta_{m}}{k_{j}}\left[\frac{1+\beta_{m}}{2}\left(\xi_{m, i, j}-\xi_{m, i, j-1}\right)\right. \\
& \left.+\frac{1-\beta_{m}}{2}\left(\xi_{m, i, j+1}-\xi_{m, i, j}\right)\right]+\frac{\sigma_{T}}{\varepsilon} \xi_{m, i, j} \\
= & \left(\frac{\sigma_{T}}{\varepsilon}-\varepsilon \sigma_{A}\right) \sum_{k} w_{k} \xi_{k, i, j}+\varepsilon Q_{x, i, j},
\end{aligned}
$$

$$
\begin{aligned}
\frac{\theta \eta_{m}}{k_{j}}\left[\frac{1+\beta_{m}}{2}\left(\psi_{m, i, j}+\chi_{m, i, j}+\psi_{m, i, j-1}+\xi_{m, i, j-1}-2 \psi_{m, i, j}\right)\right. \\
\quad+\frac{1-\beta_{m}}{2}\left(\psi_{m, i, j+1}-\chi_{m, i, j+1}\right. \\
\left.\left.\quad+\psi_{m, i, j}-\chi_{m, i, j}-2 \psi_{m, i, j}\right)\right] \\
\quad \times \frac{\gamma \mu_{m}}{h_{i}}\left[\frac{1+\alpha_{m}}{2}\left(\chi_{m, i, j}-\chi_{m, i-1, j}\right)\right. \\
\left.\quad+\frac{1-\alpha_{m}}{2}\left(\chi_{m, i+1, j}-\chi_{m, i, j}\right)\right]+\frac{\sigma_{T}}{\varepsilon} \chi_{m, i, j} \\
=\left(\frac{\sigma_{T}}{\varepsilon}-\varepsilon \sigma_{A}\right) \sum_{k} w_{k} \chi_{k, i, j}+\varepsilon Q_{y, i, j} .
\end{aligned}
$$


We now expand the dependent variables into asymptotic series as in Section 2:

$$
\begin{aligned}
& \psi_{m, i, j}=\sum_{v \geqslant 0} \psi_{m, i, j}^{(v)} \varepsilon^{v}, \\
& \xi_{m, i, j}=\sum_{v \geqslant 0} \xi_{m, i, j}^{(v)} \varepsilon^{v}, \\
& \chi_{m, i, j}=\sum_{v \geqslant 0} \chi_{m, i, j}^{(v)} \varepsilon^{v} .
\end{aligned}
$$

Inserting Eq. (45) into Eqs. (42)-(44) and equating the coefficients of $\varepsilon^{-1}$, we find the system of zeroth order equations, which expresses that to leading order, all cell-average independent variables are isotropic, i.e., independent of $m$ :

$$
\psi_{m, i, j}^{(0)}=\Psi_{i, j}^{(0)}, \quad \xi_{m, i, j}^{(0)}=\Xi_{i, j}^{(0)}, \quad \chi_{m, i, j}^{(0)}=X_{i, j}^{(0)} .
$$

Equating the coefficients of $\varepsilon^{0}$, we find the first-order equations:

$$
\begin{aligned}
\frac{\mu_{m}}{h_{i}}[ & \frac{1+\alpha_{m}}{2}\left(\Psi_{i, j}^{(0)}+\Xi_{i, j}^{(0)}-\Psi_{i-1, j}^{(0)}-\Xi_{i-1, j}^{(0)}\right) \\
& \left.+\frac{1-\alpha_{m}}{2}\left(\Psi_{i+1, j}^{(0)}-\Xi_{i+1, j}^{(0)}-\Psi_{i, j}^{(0)}+\Xi_{i, j}^{(0)}\right)\right] \\
& +\frac{\eta_{m}}{k_{j}}\left[\frac{1+\beta_{m}}{2}\left(\Psi_{i, j}^{(0)}+X_{i, j}^{(0)}-\Psi_{i, j-1}^{(0)}-X_{i, j-1}^{(0)}\right)\right. \\
& \left.+\frac{1-\beta_{m}}{2}\left(\Psi_{i, j+1}^{(0)}-X_{i, j+1}^{(0)}-\Psi_{i, j}^{(0)}+X_{i, j}^{(0)}\right)\right] \\
& +\sigma_{T} \psi_{m, i, j}^{(1)}=\sigma_{T} \sum_{k} w_{k} \psi_{k, i, j}^{(1)}, \\
\frac{\theta \mu_{m}}{h_{i}}[ & \frac{1+\alpha_{m}}{2}\left(\Psi_{i, j}^{(0)}+\Xi_{i, j}^{(0)}+\Psi_{i-1, j}^{(0)}+\Xi_{i-1, j}^{(0)}-2 \Psi_{i, j}^{(0)}\right) \\
& \left.+\frac{1-\alpha_{m}}{2}\left(\Psi_{i+1, j}^{(0)}-\Xi_{i+1, j}^{(0)}+\Psi_{i, j}^{(0)}-\Xi_{i, j}^{(0)}-2 \Psi_{i, j}^{(0)}\right)\right] \\
& +\frac{\gamma \eta_{m}}{k_{j}}\left[\frac{1+\beta_{m}}{2}\left(\Xi_{i, j}^{(0)}-\Xi_{i, j-1}^{(0)}\right)\right. \\
& \left.+\frac{1-\beta_{m}}{2}\left(\Xi_{i, j+1}^{(0)}-\Xi_{i, j}^{(0)}\right)\right] \\
& +\sigma_{T} \xi_{m, i, j}^{(1)}=\sigma_{T} \sum_{k} w_{k} \xi_{k, i, j}^{(1)},
\end{aligned}
$$$$
\frac{\theta \eta_{m}}{k_{j}}\left[\frac{1+\beta_{m}}{2}\left(\Psi_{i, j}^{(0)}+X_{i, j}^{(0)}+\Psi_{i, j}^{(0)}{ }_{1}+X_{i, j-1}^{(0)}-2 \Psi_{i, j}^{(0)}\right)\right.
$$$$
\left.+\frac{1-\beta_{m}}{2}\left(\Psi_{i, j+1}^{(0)}-X_{i, j+1}^{(0)}+\Psi_{i, j}^{(0)}-X_{i, j}^{(0)}-2 \Psi_{i, j}^{(0)}\right)\right]
$$

$$
\begin{aligned}
& +\frac{\gamma \mu_{m}}{h_{t}}\left[\frac{1+\alpha_{m}}{2}\left(X_{i, j}^{(0)}-X_{i-1, j}^{(0)}\right)\right. \\
& \left.+\frac{1-\alpha_{m}}{2}\left(X_{i+1, j}^{(0)}-X_{i, j}^{(0)}\right)\right] \\
& +\sigma_{T} \chi_{m, i, j}^{(1)}=\sigma_{T} \sum_{k} w_{k} \chi_{k, i, j}^{(1)} .
\end{aligned}
$$

We introduce the notation

$$
\rho:=\sum_{k=1}^{N} w_{k}\left|\mu_{k}\right|
$$

Equations (10) and (11) then imply

$$
\rho=\sum_{k=1}^{N} w_{k}\left|\mu_{k}\right|=\sum_{k=1}^{N} w_{k}\left|\eta_{k}\right|
$$

Multiplying each of Eqs. (46)-(48) by $w_{m}$, summing over $m$, and dividing by $\rho$, we find

$$
\begin{aligned}
\frac{1}{h_{i}}[ & \left.-\Psi_{i+1, j}^{(0)}+2 \Psi_{i, j}^{(0)}-\Psi_{i-1, j}^{(0)}+\Xi_{i+1, j}^{(0)}-\Xi_{i-1, j}^{(0)}\right] \\
& +\frac{1}{k_{j}}\left[-\Psi_{i, j+1}^{(0)}+2 \Psi_{i, j}^{(0)}-\Psi_{i, j-1}^{(0)}\right. \\
& \left.+X_{i, j+1}^{(0)}-X_{i, j-1}^{(0)}\right]=0, \\
\frac{\theta}{h_{i}}\left[-\Psi_{i+1, j}^{(0)}+\Psi_{i-1, j}^{(0)}+\Xi_{i+1, j}^{(0)}+2 \Xi_{i, j}^{(0)}+\Xi_{i-1, j}^{(0)}\right] & \\
& +\frac{\gamma}{k_{j}}\left[-\Xi_{i, j+1}^{(0)}+2 \Xi_{i, j}^{(0)}-\Xi_{i, j-1}^{(0)}\right]=0, \\
\frac{\theta}{k_{j}}[- & \left.\Psi_{i, j+1}^{(0)}+\Psi_{i, j-1}^{(0)}+X_{i, j+1}^{(0)}+2 X_{i, j}^{(0)}+X_{i, j-1}^{(0)}\right] \\
& +\frac{\gamma}{h_{i}}\left[-X_{i+1, j}^{(0)}+2 X_{i, j}^{(0)}-X_{i-1, j}^{(0)}\right]=0 .
\end{aligned}
$$

Equations (49)-(51) impose conditions on the zeroth order quantities $\Psi^{0}, \Xi^{0}$, and $X^{0}$. We shall now argue that these conditions are so restrictive that $\Psi^{0}$ cannot, in general, satisfy a consistent and stable discretization of the diffusion equation (19). Assume that $h_{i}=h$ and $k_{j}=k$ for all $i, j$. We can then study Eqs. (49)-(51) using discrete Fourier expansions. Inserting a Fourier mode of the form

$$
\left(\begin{array}{l}
\Psi_{i, j}^{(0)} \\
\Xi_{i, j}^{(0)} \\
X_{i, j}^{(0)}
\end{array}\right)=\left(\begin{array}{c}
\hat{\Psi} \\
\hat{\Xi} \\
\hat{X}
\end{array}\right) \cdot \exp \left[\sqrt{-1}\left(\frac{\lambda}{h} x_{i, j}+\frac{\omega}{k} y_{i, j}\right)\right]
$$

into Eqs. (49)-(51), we obtain a system of the form

$$
A(\lambda, \omega)\left(\begin{array}{c}
\hat{\Psi} \\
\hat{\Xi} \\
\hat{X}
\end{array}\right)=\left(\begin{array}{l}
0 \\
0 \\
0
\end{array}\right)
$$


where $A(\lambda, \omega)$ is a complex $3 \times 3$ matrix, the characteristic matrix of the system. A computation shows that

$$
\begin{aligned}
\operatorname{det} A(\lambda, \omega)= & \gamma\left[\theta \frac{(1-\cos \lambda) \sin ^{2} \omega}{h k^{2}}\right. \\
& +\gamma \frac{(1-\cos \lambda)^{2}(1-\cos \omega)}{h^{2} k} \\
& +\theta \frac{(1-\cos \omega) \sin ^{2} \lambda}{k h^{2}} \\
& \left.+\gamma \frac{\left(1-\cos (\omega)^{2}(1-\cos \lambda)\right.}{k^{2} h}\right] .
\end{aligned}
$$

Clearly, this expression is nonnegative. We always choose $\theta>0$. If also $\gamma>0$, then $\operatorname{det} A(\lambda, \omega)=0 \Leftrightarrow \lambda=0$ or $\omega=0$. We thus conclude that unless $\gamma=0$, Eqs. (46)-(48) imply restrictions on the Fourier modes that can appear in the solution, only permitting modes with either no variation or the fastest oscillation possible on the given mesh in at least one of the two coordinate directions. Thus the scheme cannot have a correct diffusion limit in general, unless $\gamma=0$. In particular, the unmodified scheme $(\theta=3, \gamma=1)$ does not have an asymptotic diffusion limit consistent with the continuous one.

\section{THE ASYMPTOTIC DIFFUSION LIMIT FOR $\theta>0$ AND $\gamma=0$}

In this section, we consider the case $\theta>0$ and $\gamma=0$. It is clear that $\gamma=0$ is not always a good choice. Consider, for example, Eqs. (40) and (41) for voids $\left(\sigma_{T}=\sigma_{A}=0\right)$ and assume that $Q_{x} \equiv 0$ and $Q_{y} \equiv 0$. Setting $\gamma=0$, one then obtains the diamond differencing scheme, which can lead to large unphysical oscillations. In practice, one would thus not use $\gamma=0$ for all problems. However, one might use a formula for $\gamma$ depending on $\varepsilon$ with the property that $\gamma \rightarrow 0$ as $\varepsilon \rightarrow 0$, on a fixed mesh. If this convergence is sufficiently fast $\left(\gamma=O\left(\varepsilon^{2}\right)\right.$ is enough), the analysis given in the present section still applies.

We recall our convention that subscripts of dependent variables are evaluated modulo $I$ or modulo $J$, i.e., $\psi_{m, i+1, j}=\psi_{m, i, j+J}=\psi_{m, i, j}$, etc. We consider the following equations:

$$
\begin{aligned}
\frac{\mu_{m}}{h_{i}}\left(\psi_{m, i}+1 / 2, j\right. & \left.-\psi_{m, i-1 / 2, j}\right) \\
& +\frac{\eta_{m}}{k_{j}}\left(\psi_{m, i, j+1 / 2}-\psi_{m, i, j-1 / 2}\right)+\frac{\sigma_{T}}{\varepsilon} \psi_{m, i, j} \\
= & \left(\frac{\sigma_{T}}{\varepsilon}-\varepsilon \sigma_{A}\right) \sum_{k=1}^{N} w_{k} \psi_{k, i, j}+\varepsilon Q_{i, j},
\end{aligned}
$$

$$
\begin{gathered}
\frac{\theta \mu_{m}}{h_{i}}\left(\psi_{m, i+1 / 2, j}+\psi_{m, i-1 / 2, j}-2 \psi_{m, i, j}\right)+\frac{\sigma_{T}}{\varepsilon} \xi_{m, i, j} \\
=\left(\frac{\sigma_{T}}{\varepsilon}-\varepsilon \sigma_{A}\right) \sum_{k=1}^{N} w_{k} \xi_{k, i, j}+\varepsilon Q_{x, i, j}, \\
\frac{\theta \eta_{m}}{k_{j}}\left(\psi_{m, i, j+1 / 2}+\psi_{m, i, j-1 / 2}-2 \psi_{m, i, j}\right)+\frac{\sigma_{T}}{\varepsilon} \chi_{m, i, j} \\
=\left(\frac{\sigma_{T}}{\varepsilon}-\varepsilon \sigma_{A}\right) \sum_{k=1}^{N} w_{k} \chi_{k, i, j}+\varepsilon Q_{y, i, j} .
\end{gathered}
$$

The system is completed by Eqs. (30) and (31).

In Section 4, the first step was to eliminate the cell edge unknowns. This led to a relatively simple calculation showing that there is no correct asymptotic diffusion limit for the scheme with $\gamma>0$. Consideration of the zeroth and first-order equations was sufficient for this argument.

In the present context, we have found it more convenient not to eliminate the cell edge unknowns. As previously, we consider asymptotic expansions of the form

$$
\begin{aligned}
& \psi_{m}=\sum_{v \geqslant 0} \psi_{m}^{(v)} \varepsilon^{v}, \\
& \xi_{m}=\sum_{v \geqslant 0} \xi_{m}^{(v)} \varepsilon^{v}, \\
& \chi_{m}=\sum_{v \geqslant 0} \chi_{m}^{(v)} \varepsilon^{v} .
\end{aligned}
$$

Inserting these expansions into Eqs. (53)-(55), we see as before that, to leading order, all cell center unknowns are isotropic, i.e., independent of $m$ :

$$
\psi_{m, i, j}^{(0)}=\Psi_{i, j}^{(0)}, \quad \xi_{m, i, j}^{(0)}=\Xi_{i, j}^{(0)}, \quad \chi_{m, i, j}^{(0)}=X_{i, j}^{(0)}
$$

At this point, we do not obtain isotropy for the cell edge unknowns. However, we shall see later $\psi_{m, i \pm 1 / 2, j}^{(0)}$ and $\psi_{m, i, j \pm 1 / 2}^{(0)}$ are isotropic as well.

We proceed to the first-order equations:

$$
\begin{gathered}
\sigma_{T}\left(\psi_{m, i, j}^{(1)}-\sum_{k=1}^{N} w_{k} \psi_{k, i, j}^{(1)}\right) \\
=-\frac{\mu_{m}}{h_{i}}\left(\psi_{m, i+1 / 2, j}^{(0)}-\psi_{m, i-1 / 2, j}^{(0)}\right) \\
-\frac{\eta_{m}}{k_{j}}\left(\psi_{m, i, j+1 / 2}^{(0)}-\psi_{m, i, j-1 / 2}^{(0)}\right), \\
\sigma_{T}\left(\xi_{m, i, j}^{(1)}-\sum_{k=1}^{N} w_{k} \xi_{k, i, j}^{(1)}\right) \\
=-\frac{\theta \mu_{m}}{h_{i}}\left(\psi_{m, i+1 / 2, j}^{(0)}+\psi_{m, i-1 / 2, j}^{(0)}-2 \Psi_{i, j}^{(0)}\right),
\end{gathered}
$$




$$
\begin{aligned}
\sigma_{T}\left(\chi_{m, i, j}^{(1)}-\sum_{k=1}^{N} w_{k} \chi_{k, i, j}^{(1)}\right) & \\
= & -\frac{\theta \eta_{m}}{k_{j}}\left(\psi_{m, i, j+1 / 2}^{(0)}+\psi_{m, i, j-1 / 2}^{(0)}-2 \Psi_{i, j}^{(0)}\right) \\
\psi_{m, i+1 / 2, j}^{(0)} & \\
= & \frac{1+\alpha_{m}}{2}\left(\Psi_{i, j}^{(0)}+\Xi_{i, j}^{(0)}\right) \\
& +\frac{1-\alpha_{m}}{2}\left(\Psi_{i+1, j}^{(0)}-\Xi_{i+1, j}^{(0)}\right) \\
\psi_{m, i, j+}^{(0)}+1 / 2 & \\
= & \frac{1+\beta_{m}}{2}\left(\Psi_{i, j}^{(0)}+X_{i, j}^{(0)}\right) \\
& +\frac{1-\beta_{m}}{2}\left(\Psi_{i, j+1}^{(0)}-X_{i, j+1}^{(0)}\right) .
\end{aligned}
$$

Following the strategy outlined at the end of Section 2, we use Eqs. (60)-(62) to express anisotropic first-order quantities in terms of zeroth-order quantities and isotropic first-order quantities. Thus we define

$$
\begin{aligned}
& \Psi_{i, j}^{(1)}:=\sum_{k=1}^{N} w_{k} \psi_{k, i, j}^{(1)}, \\
& \Xi_{i, j}^{(1)}:=\sum_{k=1}^{N} w_{k} \xi_{k, i, j}^{(1)}, \\
& X_{i, j}^{(1)}:=\sum_{k=1}^{N} w_{k} \chi_{k, i, j}^{(1)} .
\end{aligned}
$$

Then Eqs. (60)-(62) yield

$$
\begin{aligned}
\psi_{m, i, j}^{(1)}= & \Psi_{i, j}^{(1)}-\frac{\mu_{m}}{\sigma_{T} h_{i}}\left(\psi_{m, i+1 / 2, j}^{(0)}-\psi_{m, i-1 / 2, j}^{(0)}\right) \\
& -\frac{\eta_{m}}{\sigma_{T} k_{j}}\left(\psi_{m, i, j+1 / 2}^{(0)}-\psi_{m, i, j-1 / 2}^{(0)}\right), \\
\xi_{m, i, j}^{(1)}= & \Xi_{i, j}^{(1)}-\frac{\theta \mu_{m}}{\sigma_{T} h_{i}}\left(\psi_{m, i+1 / 2, j}^{(0)}\right. \\
& \left.+\psi_{m, i-1 / 2, j}^{(0)}-2 \Psi_{i, j}^{(0)}\right), \\
\chi_{m, i, j}^{(1)}= & X_{i, j}^{(1)}-\frac{\theta \eta_{m}}{\sigma_{T} k_{j}}\left(\psi_{m, i, j+1 / 2}^{(0)}\right. \\
& \left.+\psi_{m, i, j-1 / 2}^{(0)}-2 \Psi_{i, j}^{(0)}\right) .
\end{aligned}
$$

The second-order equations arising from Eqs. (53) (55) are

$$
\begin{array}{r}
\sigma_{T}\left(\psi_{m, i, j}^{(2)}-\sum_{k=1}^{N} w_{k} \psi_{k, i, j}^{(2)}\right) \\
=-\frac{\mu_{m}}{h_{i}}\left(\psi_{m, i+1 / 2, j}^{(1)}-\psi_{m, i-1 / 2, j}^{(1)}\right) \\
-\frac{\eta_{m}}{k_{j}}\left(\psi_{m, i, j+1 / 2}^{(1)}-\psi_{m, i, j-1 / 2}^{(1)}\right) \\
-\sigma_{A} \Psi_{i, j}^{(0)}+Q_{i, j}, \\
\sigma_{T}\left(\xi_{m, i, j}^{(2)}-\sum_{k=1}^{N} w_{k} \xi_{k, i, j}^{(2)}\right) \\
=-\frac{\theta \mu_{m}}{h_{i}}\left(\psi_{m, i+1 / 2, j}^{(1)}+\psi_{i-1 / 2, j}^{(1)}\right. \\
\left.-2 \psi_{m, i, j}^{(1)}\right)-\sigma_{A} \Xi_{i, j}^{(0)}+Q_{x, i, j}, \\
\sigma_{T}\left(\chi_{m, i, j}^{(2)}-\sum_{k=1}^{N} w_{k} \chi_{k, i, j}^{(2)}\right) \\
=-\frac{\theta \eta_{m}}{k_{j}}\left(\psi_{m, i, j+1 / 2}^{(1)}+\psi_{m, i, j-1 / 2}^{(1)}\right. \\
\left.-2 \psi_{m, i, j}^{(1)}\right)-\sigma_{A} X_{i, j}^{(0)}+Q_{y, i, j} .
\end{array}
$$

Multiplying these equations by $w_{m}$ and summing over $m$, we obtain solvability conditions. To express these conditions in a brief form, we define

$$
\begin{aligned}
J_{\mu, i, j}^{(1)} & :=\sum_{k=1}^{N} w_{k} \mu_{k} \psi_{k, i, j}^{(1)}, \\
J_{\eta, i, j}^{(1)} & :=\sum_{k=1}^{N} w_{k} \eta_{k} \psi_{k, i, j}^{(1)}, \\
J_{i \pm 1 / 2, j}^{(1)} & :=\sum_{k=1}^{N} w_{k} \mu_{k} \psi_{k, i \pm 1 / 2, j}^{(1)}, \\
J_{i, j \pm 1 / 2}^{(1)} & :-\sum_{k=1}^{N} w_{k} \eta_{k} \psi_{k, i, j \pm 1 / 2}^{(1)} .
\end{aligned}
$$

These variables are called currents. They give the net rate at which particles cross cells and cell boundaries in the horizontal and vertical directions. With this notation, the solvability conditions become

$$
\begin{aligned}
& k_{j}\left(J_{i+1 / 2, j}^{(1)}-J_{i-1 / 2, j}^{(1)}\right)+h_{i}\left(J_{i, j+1 / 2}^{(1)}-J_{i, j-1 / 2}^{(1)}\right) \\
& \quad=h_{i} k_{j}\left(Q_{i, j}-\sigma_{A} \Psi_{i, j}^{(0)}\right) \\
& \theta k_{j}\left(J_{i+1 / 2, j}^{(1)}+J_{i-1 / 2, j}^{(1)}\right) \\
& \quad=2 \theta k_{j} J_{\mu, i, j}^{(1)}+h_{i} k_{j}\left(Q_{x, i, j}-\sigma_{A} \Xi_{i, j}^{(0)}\right), \\
& \theta h_{i}\left(J_{i, j+1 / 2}^{(1)}+J_{i, j-1 / 2}^{(1)}\right) \\
& \quad=2 \theta h_{i} J_{\eta, i, j}^{(1)}+h_{i} k_{j}\left(Q_{y, i, j}-\sigma_{A} X_{i, j}^{(0)}\right) .
\end{aligned}
$$


Equations (78)-(80) can be simplified if we observe that the cell edge currents, i.e., the variables $J_{i \pm 1 / 2, j}^{(1)}$ and $J_{i, j \pm 1 / 2}^{(1)}$, can be eliminated by the following procedure. First, we form the equation $(78)_{i, j}+(78)_{i+1, j}+(78)_{i, j+1}+(78)_{i+1, j+1}$ :

$$
\begin{aligned}
k_{j}\left(J_{i+3 / 2, j}^{(1)}-J_{i-1 / 2, j}^{(1)}\right) \\
\quad+k_{j+1}\left(J_{i+3 / 2, j+1}^{(1)}-J_{i-1 ; 2, j+1}^{(1)}\right) \\
\quad+h_{i}\left(J_{i, j+3 / 2}^{(1)}-J_{i, j-1 / 2}^{(1)}\right) \\
\quad+h_{i+1}\left(J_{i+1, j+3 / 2}^{(1)}-J_{i+1, j-1 / 2}^{(1)}\right) \\
\quad+h_{i} k_{j}\left(Q_{i, j}-\sigma_{A} \Psi_{i, j}^{(0)}\right) \\
\quad+h_{i+1} k_{i}\left(Q_{i+1, j}-\sigma_{A} \Psi_{i+1, j}^{(0)}\right) \\
=h_{i} k_{j+1}\left(Q_{i, j+1}-\sigma_{A} \Psi_{i, j+1}^{(0)}\right) \\
\quad+h_{i+1} k_{j+1}\left(Q_{i+1, j+1}-\sigma_{A} \Psi_{i+1, j+1}^{(0)}\right)
\end{aligned}
$$

Similarly, we form $-(79)_{i, j}+(79)_{i+1, j}-(79)_{i, j+1}+$ $(79)_{i+1, j+1}$, and divide by $\theta$ :

$$
\begin{aligned}
k_{j}\left(J_{i+3 / 2, j}^{(1)}-J_{i-1 / 2, j}^{(1)}\right) & \\
& +k_{j+1}\left(J_{i+3 / 2, j+1}^{(1)}-J_{i-1 / 2, j+1}^{(1)}\right) \\
= & -2 k_{j} J_{\mu, i, j}^{(1)}-\frac{h_{i} k_{j}}{\theta}\left(Q_{x, i, j}-\sigma A \Xi_{i, j}^{(0)}\right) \\
& +2 k_{j} J_{\mu, i+1, j}^{(1)}+\frac{h_{i+1} k_{j}}{\theta} \\
& \times\left(Q_{x, i+1, j}-\sigma_{A} \Xi_{i+1, j}^{(0)}\right) \\
& -2 k_{j+1} J_{\mu, i, j+1}^{(1)}-\frac{h_{i} k_{j+1}}{\theta} \\
& \times\left(Q_{x, i, j+1}-\sigma_{A} \Xi_{i, j+1}^{(0)}\right) \\
& +2 k_{j+1} J_{\mu, i+1, j+1}^{(1)}+\frac{h_{i+1} k_{j+1}}{\theta} \\
& \times\left(Q_{x, i+1, j+1}-\sigma_{A} \Xi_{i+1, j+1}^{(0)}\right) .
\end{aligned}
$$

We also form $-(80)_{i, j}-(80)_{i+1, j}+(80)_{i, j+1}+(80)_{i+1, j+1}$, and divide by $\theta$ :

$$
\begin{aligned}
h_{i}\left(J_{i, j+3 / 2}^{(1)}-J_{i, j-1 / 2}^{(1)}\right) & \\
& +h_{i+1}\left(J_{i+1, j+3 / 2}^{(1)}-J_{i+1, j-1 / 2}^{(1)}\right) \\
= & -2 h_{i} J_{\eta, i, j}^{(1)}-\frac{h_{i} k_{j}}{\theta}\left(Q_{y, i, j}-\sigma_{A} X_{i, j}^{(0)}\right) \\
& +2 h_{i} J_{\eta, i, j+1}^{(1)}+\frac{h_{i} k_{j+1}}{\theta} \\
& \times\left(Q_{y, i, j+1}-\sigma_{A} X_{i, j+1}^{(0)}\right) \\
& -2 h_{i+1} J_{\eta, i+1, j}^{(1)}-\frac{h_{i+1} k_{j}}{\theta}
\end{aligned}
$$

$$
\begin{aligned}
& \times\left(Q_{y, i+1, j}-\sigma_{A} X_{i+1, j}^{(0)}\right) \\
& +2 h_{i+1} J_{\eta, i+1, j+1}^{(1)}+\frac{h_{i+1} k_{j+1}}{\theta} \\
& \times\left(Q_{y, i+1, j+1}-\sigma_{A} X_{i+1, j+1}^{(0)}\right) .
\end{aligned}
$$

If we now subtract Eqs. (82) and (83) from Eq. (81), the cell-edge currents are eliminated, and we obtain

$$
\begin{aligned}
2 k_{j+1}( & \left.J_{\mu, i+1, j+1}^{(1)}-J_{\mu, i, j+1}^{\{1)}\right) \\
& +2 k_{j}\left(J_{\mu, i+1, j}^{(1)}-J_{\mu, i, j}^{(1)}\right) \\
& +2 h_{i+1}\left(J_{\eta, i+1, j+1}^{(1)}-J_{\eta, i+1, j}^{(1)}\right) \\
& +2 h_{i}\left(J_{\eta, i, j+1}^{(1)}-J_{\eta, i, j}^{(1)}\right) \\
& +h_{i} k_{j} \sigma_{A}\left(\Psi_{i, j}^{(0)}+\frac{\Xi_{i, j}^{(0)}+X_{i, j}^{(0)}}{\theta}\right) \\
& +h_{i+1} k_{j} \sigma_{A}\left(\Psi_{i+1, j}^{(0)}-\frac{\Xi_{i+1, j}^{(0)}-X_{i+1, j}^{(0)}}{\theta}\right) \\
& +h_{i} k_{j+1} \sigma_{A}\left(\Psi_{i, j+1}^{(0)}+\frac{\Xi_{j, j+1}^{(0)}-X_{i, j+1}^{(0)}}{\theta}\right) \\
& +h_{i+1} k_{j+1} \sigma_{A,}\left(\Psi_{i+1, j+1}^{(0)}-\frac{\Xi_{i+1, j+1}^{(0)}+X_{i+1, j+1}^{(0)}}{\theta}\right) \\
= & (h k Q)_{i+1 / 2, j+1 / 2},
\end{aligned}
$$

with

$$
\begin{aligned}
(h k Q)_{i+1 / 2, j+1 / 2} & =h_{i} k_{j}\left(Q_{i, j}+\frac{Q_{x, i, j}+Q_{y, i, j}}{\theta}\right) \\
& +h_{i+1} k_{j}\left(Q_{i+1, j}-\frac{Q_{x, i+1, j}-Q_{y, i+1, j}}{\theta}\right) \\
& +h_{i} k_{j+1}\left(Q_{i, j+1}+\frac{Q_{x, i, j+1}-Q_{y, i, j+1}}{\theta}\right) \\
& +h_{i+1} k_{j+1}\left(Q_{i+1, j+1}-\frac{Q_{x, i+1, j+1}+Q_{y, i+1, j+1}}{\theta}\right) .
\end{aligned}
$$

Our next goal is to remove the first-order currents from Eq. (84), using Eq. (68). An undesirable feature of Eq. (68) is that it contains the anisotropic zeroth-order quantities $\psi_{m, i+1 / 2, j}^{(0)}$ and $\psi_{m, i, j+1 / 2}^{(0)}$. Before using Eq. (68) in Eq. (84), we shall therfore examine $\psi_{m, i \pm 1 / 2, j}^{(0)}$ and $\psi_{m, i, j \pm 1 / 2}^{(0)}$ more closely and derive expressions for them showing that they are "almost isotropic." It is clear that a result of this kind will follow from Eqs. (63) and (64).

In this context, it is natural to introduce the notation

$$
\Psi_{i \pm 1 / 2, j}^{(0)}:=\sum_{k=1}^{N} w_{k} \psi_{k, i \pm 1 / 2, j}^{(0)}
$$


and

$$
\Psi_{i, j \pm 1 / 2}^{(0)}:=\sum_{k=1}^{N} w_{k} \psi_{k, i, j \pm 1 / 2}^{(0)}
$$

It will soon become apparent that it is also useful to introduce the zeroth-order currents

$$
J_{i \pm 1 / 2, j}^{(0)}:=\sum_{k=1}^{N} w_{k} \mu_{k} \psi_{k, i \pm 1 / 2, j}^{(0)}
$$

and

$$
J_{i, j \pm 1 / 2}^{(0)}:=\sum_{k=1}^{N} w_{k} \eta_{k} \psi_{k, i, j \pm 1 / 2}^{(0)}
$$

With this notation, Eq. (63) yields, upon operating by $\sum_{k=1}^{N} w_{k}(\cdot)$ and $\sum_{k=1}^{N} w_{k} \mu_{k}(\cdot)$, the equations

$$
\Psi_{i+1 / 2, j}^{(0)}=\frac{1}{2}\left[\left(\Psi_{i, j}^{(0)}+\Xi_{i, j}^{(0)}\right)+\left(\Psi_{i+1, j}^{(0)}-\Xi_{i+1, j}^{(0)}\right)\right]
$$

and

$$
J_{i+1 / 2, j}^{(0)}=\frac{\rho}{2}\left[\left(\Psi_{i, j}^{(0)}+\Xi_{i, j}^{(0)}\right)-\left(\Psi_{i+1, j}^{(0)}-\Xi_{i+1, j}^{(0)}\right)\right] .
$$

Here we have defined, as in Section 4,

$$
\rho:=\sum_{k=1}^{N} w_{k}\left|\mu_{k}\right|=\sum_{k=1}^{N} w_{k}\left|\eta_{k}\right|
$$

Similarly, Eq. (64) yields

$$
\Psi_{i, j+1 / 2}^{(0)}=\frac{1}{2}\left[\left(\Psi_{i, j}^{(0)}+X_{i, j}^{(0)}\right)+\left(\Psi_{i, j+1}^{(0)}-X_{i, j+1}^{(0)}\right)\right]
$$

and

$$
J_{i, j+1 / 2}^{(0)}=\frac{\rho}{2}\left[\left(\Psi_{i, j}^{(0)}+X_{i, j}^{(0)}\right)-\left(\Psi_{i, j+1}^{(0)}-X_{i, j+1}^{(0)}\right)\right] .
$$

Inserting Eqs. (90)-(94) back into Eqs. (63) and (64), we find

$$
\psi_{m, i+1 / 2, j}^{(0)}=\Psi_{i+1 / 2, j}^{(0)}+\frac{\alpha_{m}}{\rho} J_{i+1 / 2, j}^{(0)}
$$

and

$$
\psi_{m, i, j+1 / 2}^{(0)}=\Psi_{i, j+1 / 2}^{(0)}+\frac{\beta_{m}}{\rho} J_{i, j+1 / 2}^{(0)} .
$$

Thus, the $\psi_{m, i+1 / 2, j}^{(0)}$ and $\psi_{m, i, j+1 / 2}^{(0)}$ depend only on the signs of $\mu_{m}$ and $\eta_{m}$, not their values.

We insert Eqs. (95) and (96) into Eq. (68), multiply by $w_{m} \mu_{m}$, and sum over $m$. The result is

$$
J_{\mu, i, j}^{(1)}=-\frac{D}{h_{i}}\left(\Psi_{i+1 / 2, j}^{(0)}-\Psi_{i-1 / 2, j}^{(0)}\right)
$$

with

$$
D:=\frac{1}{3 \sigma_{T}} .
$$

Here we have used our assumptions on the quadrature scheme, Eqs. (5)-(11). An analogous argument, with the roles of the two coordinate directions reversed, gives

$$
J_{\eta, i, j}^{(1)}=-\frac{D}{k_{j}}\left(\Psi_{i, j+1 / 2}^{(0)}-\Psi_{i, j-1 / 2}^{(0)}\right) .
$$

Inserting Eqs. (97) and (99) into Eq. (84), we are left with an equation involving zeroth-order isotropic quantities only. This equation contains cell variables and edge variables. We shall now eliminate the cell variables.

Equations (90) and (91) can be solved for $\Psi_{i, j}^{(0)}+\Xi_{i, j}^{(0)}$ and $\Psi_{i+1, j}^{(0)}-\Xi_{i+1, j}^{(0)}$, and therefore for $\Psi_{i, j}^{(0)}$ and $\Xi_{i, j}^{i, j}$,

$$
\begin{aligned}
\Psi_{i, j}^{(0)}= & \frac{1}{2}\left[\left(\Psi_{i+1 / 2, j}^{(0)}+\Psi_{i-1 / 2, j}^{(0)}\right)\right. \\
& \left.+\frac{1}{\rho}\left(J_{i+1 / 2, j}^{(0)}-J_{i-1 / 2, j}^{(0)}\right)\right]
\end{aligned}
$$

and

$$
\begin{aligned}
\Xi_{i, j}^{(0)}= & \frac{1}{2}\left[\left(\Psi_{i+1 / 2, j}^{(0)}-\Psi_{i-1 / 2, j}^{(0)}\right)\right. \\
& \left.+\frac{1}{\rho}\left(J_{i+1 / 2, j}^{(0)}+J_{i-1 / 2, j}^{(0)}\right)\right] .
\end{aligned}
$$

A similar calculation, with the roles of the coordinates reversed, gives

$$
\begin{aligned}
\Psi_{i, j}^{(0)}= & \frac{1}{2}\left[\left(\Psi_{i, j+1 / 2}^{(0)}+\Psi_{i, j-1 / 2}^{(0)}\right)\right. \\
& \left.+\frac{1}{\rho}\left(J_{i, j+1 / 2}^{(0)}-J_{i, j-1 / 2}^{(0)}\right)\right]
\end{aligned}
$$

and

$$
\begin{aligned}
X_{i, j}^{(0)}= & \frac{1}{2}\left[\left(\Psi_{i, j+1 / 2}^{(0)}-\Psi_{i, j-1 / 2}^{(0)}\right)\right. \\
& \left.+\frac{1}{\rho}\left(J_{i, j+1 / 2}^{(0)}+J_{i, j-1 / 2}^{(0)}\right)\right] .
\end{aligned}
$$

We note that Eqs. (100) and (102) imply the consistency condition

$$
\begin{aligned}
& \left(\Psi_{i+1 / 2, j}^{(0)}+\Psi_{i-1 / 2, j}^{(0)}\right)+\frac{1}{\rho}\left(J_{i+1 / 2, j}^{(0)}-J_{i-1 / 2, j}^{(0)}\right) \\
& =\left(\Psi_{i, j+1 / 2}^{(0)}+\Psi_{i, j-1 / 2}^{(0)}\right) \\
& \quad+\frac{1}{\rho}\left(J_{i, j+1 / 2}^{(0)}-J_{i, j-1 / 2}^{(0)}\right) .
\end{aligned}
$$

We shall make use of this equation later. 
If we insert Eqs. (97) and (99) into Eq. (84), and then insert Eqs. (100)-(103) into the result, we obtain an equation involving $\Psi_{i \pm 1 / 2, j}^{(0)}, \Psi_{i, j \pm 1 / 2}^{(0)}, J_{i \pm 1 / 2, j}^{(0)}$, and $J_{i, j \pm 1 / 2}^{(0)}$. (We note that there is a slight ambiguity: Should one use Eq. (100) or Eq. (102)? However, the final result will be independent of this choice.)

Next we shall show that the currents $J_{i \pm 1 / 2, j}^{(0)}$ and $J_{i, j \pm 1 / 2}^{(0)}$ are zero. This follows from the solvability conditions for the first-order equations (68)-(70). Those equations are

$$
\begin{array}{r}
\frac{J_{i+1 / 2, j}^{(0)}-J_{i-1 / 2, j}^{(0)}+\frac{J_{i, j+1 / 2}^{(0)}-J_{i, j-1 / 2}^{(0)}}{h_{i}}}{k_{j}}=0, \\
J_{i+1 / 2, j}^{(0)}+J_{i-1 / 2, j}^{(0)}=0, \\
J_{i, j+1 / 2}^{(0)}+J_{i, j-1 / 2}^{(0)}=0 .
\end{array}
$$

Equation (106) implies

$$
J_{i+1 / 2, j}^{(0)}=(-1)^{i} a_{j}
$$

for constants $a_{j}$ independent of $i$. Similarly, Eq. (107) implies

$$
J_{i, j+1 / 2}^{(0)}=(-1)^{j} b_{i}
$$

for constants $b_{i}$ independent of $j$. Inserting these two equations into Eq. (105), we conclude that

$$
J_{i+1 / 2, j}^{(0)}=\frac{(-1)^{i+j}}{k_{j}} A^{(0)}
$$

and

$$
J_{i, j+1 / 2}^{(0)}=\frac{(-1)^{i+j+1}}{h_{i}} A^{(0)},
$$

with a constant $A^{(0)}$ independent of $i$ and $j$. To determine $A^{(0)}$, we insert Eqs. (110) and (111) into (104):

$$
\begin{gathered}
\Psi_{i+1 / 2, j}^{(0)}+\Psi_{i-1 / 2, j}^{(0)}-\Psi_{i, j+1 / 2}^{(0)}-\Psi_{i, j-1 / 2}^{(0)} \\
=-\frac{2}{\rho}\left(\frac{1}{h_{i}}+\frac{1}{k_{j}}\right)(-1)^{i+j} A^{(0)}
\end{gathered}
$$

Multiplying this equation by $(-1)^{i+j}$, summing over $i$ and $j$ with $1 \leqslant i \leqslant 2 I, 1 \leqslant j \leqslant 2 J$, and using the periodicity, we obtain $A^{(0)}=0$.

We shall now state the results derived so far in an explicit form. We insert Eqs. (97) and (99) into Eq. (84). Then we insert Eqs. (100), (101), and (103) into the result and use that $J_{i \pm 1 / 2, j}^{(0)}$ and $J_{i, j \pm 1 / 2}^{(0)}$ are zero. We obtain the following equation:

$$
\begin{aligned}
& 2\left[k _ { j + 1 } \left(-\frac{D}{h_{i+1}}\left(\Psi_{i+3 / 2, j+1}-\Psi_{i+1 / 2, j+1}\right)\right.\right. \\
& \left.+\frac{D}{h_{i}}\left(\Psi_{i+1 / 2, j+1}-\Psi_{i-1 / 2, j+1}\right)\right) \\
& +k_{j}\left(-\frac{D}{h_{i+1}}\left(\Psi_{i+3 / 2, i}-\Psi_{i+1 / 2, j}\right)\right. \\
& \left.\left.+\frac{D}{h_{i}}\left(\Psi_{i+1 / 2, j}-\Psi_{i-1 / 2, j}\right)\right)\right] \\
& +2\left[h _ { i + 1 } \left(-\frac{D}{k_{j+1}}\left(\Psi_{i+1, j+3 / 2}-\Psi_{i+1, j+1 / 2}\right)\right.\right. \\
& \left.+\frac{D}{k_{j}}\left(\Psi_{i+1, j+1 / 2}-\Psi_{i+1, j-1 / 2}\right)\right) \\
& +h_{i}\left(-\frac{D}{k_{j+1}}\left(\Psi_{i, j+3 / 2}-\Psi_{i, j+1 / 2}\right)\right. \\
& \left.\left.+\frac{D}{k_{j}}\left(\Psi_{i, j+1 / 2}-\Psi_{i, j-1 / 2}\right)\right)\right] \\
& +h_{i} k_{j} \sigma_{A}\left[\frac{1}{2}\left(\Psi_{i+1 / 2, j}+\Psi_{i-1 / 2, j}\right)\right. \\
& \left.+\frac{1}{2 \theta}\left(\Psi_{i+1 / 2, j}-\Psi_{i-1 / 2, j}+\Psi_{i, j+1 / 2}-\Psi_{i, j-1 / 2}\right)\right] \\
& +h_{i+1} k_{j} \sigma_{A}\left[\frac{1}{2}\left(\Psi_{i+3 / 2, j}+\Psi_{i+1 / 2, j}\right)\right. \\
& -\frac{1}{2 \theta}\left(\Psi_{i+3 / 2, j}-\Psi_{i+1 / 2, j}\right. \\
& \left.\left.-\Psi_{i+1, j+1 / 2}+\Psi_{i+1, j-1 / 2}\right)\right] \\
& +h_{i} k_{j+1} \sigma_{A}\left[\frac{1}{2}\left(\Psi_{i+1 / 2, j+1}+\Psi_{i-1 / 2, j+1}\right)\right. \\
& +\frac{1}{2 \theta}\left(\Psi_{i+1 / 2, j+1}-\Psi_{i-1 / 2, j+1}\right. \\
& \left.\left.-\Psi_{i, j+3 / 2}+\Psi_{i, j+1 / 2}\right)\right] \\
& +h_{i+1} k_{j+1} \sigma_{A}\left[\frac{1}{2}\left(\Psi_{i+3 / 2, j+1}+\Psi_{i+1 / 2, j+1}\right)\right. \\
& -\frac{1}{2 \theta}\left(\Psi_{i+3 / 2, j+1}-\Psi_{i+1 / 2, j+1}\right. \\
& \left.\left.+\Psi_{i+1, j+3 / 2}-\Psi_{i+1, j+1 / 2}\right)\right] \\
& =(h k Q)_{i+1 / 2, j+1 / 2} \text {. }
\end{aligned}
$$


In addition to Eq. (113), we have the consistency condition (104), which can now be written as

$$
\Psi_{i+1 / 2, j}+\Psi_{i-1 / 2, j}=\Psi_{i, j+1 / 2}+\Psi_{i, j-1 / 2}
$$

The general solution of Eq. (114) is

$$
\Psi_{i+1 / 2, j}=\frac{1}{2}\left(\Psi_{i+1 / 2, j-1 / 2}+\Psi_{i+1 / 2, j+1 / 2}\right)
$$

and

$$
\Psi_{i, j+1 / 2}=\frac{1}{2}\left(\Psi_{i-1 / 2, j+1 / 2}+\Psi_{i+1 / 2, j+1 / 2}\right)
$$

Here $\Psi_{i+1 / 2, j+1 / 2}$ is an arbitrary function defined at the cell vertices. Inserting Eqs. (115) and (116) into Eq. (113), we obtain a discrete diffusion equation for the variables $\Psi_{i+1 / 2, j+1 / 2}$, which is a consistent discretization of the continuous diffusion limit for all $\theta>0$. Because of Eq. (114), we would have obtained the same result if we had used Fq. (102) instead of Eq. (100).

For illustration, we describe this discretization for the case when $h_{i}=k_{j}=h$ for all $i$ and $j$. We then obtain

$$
\begin{aligned}
\frac{D}{2 h^{2}}\left[\begin{array}{rrr}
-1 & 0 & -1 \\
0 & 4 & 0 \\
-1 & 0 & -1
\end{array}\right] \Psi \\
\quad+\frac{\sigma_{A}}{16}\left[\begin{array}{ccc}
1-2 / \theta & 2 & 1-2 / \theta \\
2 & 4+8 / \theta & 2 \\
1-2 / \theta & 2 & 1-2 / \theta
\end{array}\right] \Psi \\
=\frac{1}{4}\left[\begin{array}{rr}
1 & 1 \\
1 & 1
\end{array}\right] Q+\frac{1}{4 \theta}\left[\begin{array}{ll}
1 & -1 \\
1 & -1
\end{array}\right] Q_{x} \\
+\frac{1}{4 \theta}\left[\begin{array}{rr}
-1 & -1 \\
1 & 1
\end{array}\right] Q_{y} .
\end{aligned}
$$

Here

$$
\begin{aligned}
& {\left[\begin{array}{rrr}
-1 & 0 & -1 \\
0 & 4 & 0 \\
-1 & 0 & -1
\end{array}\right] \Psi} \\
& :=4 \psi_{i+1 / 2, j+1 / 2} \\
& \quad-\left(\psi_{i-1 / 2, j-1 / 2}+\psi_{i-1 / 2, j+3 / 2}\right. \\
& \left.\quad+\psi_{i+3 / 2, j-1 / 2}+\psi_{i+3 / 2, j+3 / 2}\right), \\
& {\left[\begin{array}{rr}
1 & 1 \\
1 & 1
\end{array}\right] Q} \\
& \quad:=Q_{i, j}+Q_{i+1, j}+Q_{i, j+1}+Q_{i+1, j+1},
\end{aligned}
$$

etc. Equation (117) is a second-order consistent discretization of the continuous diffusion equation (19).
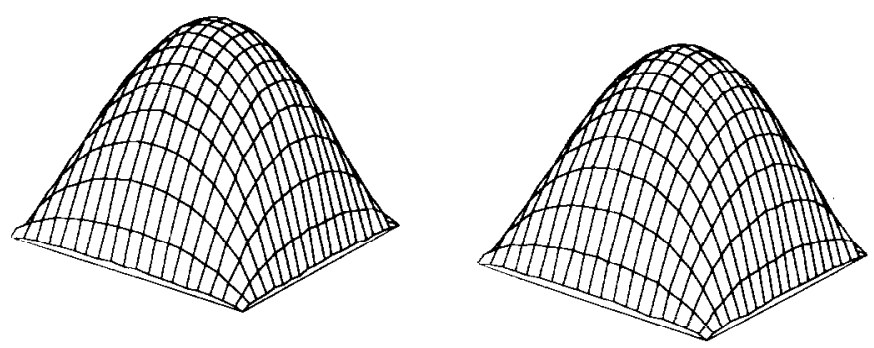

FIG. 1. $\varepsilon=10^{-2}, \operatorname{LD}$ (left) and $\operatorname{MLD}(\theta=1, \gamma=0)$ (right).

We have concluded that the MLD scheme satisfies the minimal requirement that the limits $\varepsilon \rightarrow 0$ and $h \rightarrow 0$ commute. We emphasize, however, that even Eq. (117) is not completely satisfactory: It is an inaccurate discretization of Eq. (19) if $\sigma_{A} \sigma_{T} h^{2} \gg 1$. The term that causes the problem is

$$
\left[\begin{array}{ccc}
1-2 / \theta & 2 & 1-2 / \theta \\
2 & 4+8 / \theta & 2 \\
1-2 / \theta & 2 & 1-2 / \theta
\end{array}\right] \Psi .
$$

We note that the unusual discretization of the Laplace operator in Eq. (117) does not cause any accuracy problems. The discretization is accurate if $\sigma_{A} \sigma_{T} h^{2}$ is small.

\section{A NUMERICAL EXPERIMENT}

In this section, we present a numerical experiment confirming our results. Since we had computer codes for problems on bounded domains with vacuum boundary conditions available to us, we have carried out the calculations for such problems rather than periodic problems. Thus we consider

$$
\begin{gathered}
(\boldsymbol{\Omega} \cdot \boldsymbol{\nabla}) \psi(\mathbf{x}, \boldsymbol{\Omega})+\frac{1}{\varepsilon} \psi(\mathbf{x}, \boldsymbol{\Omega}) \\
=\frac{1 / \varepsilon-\varepsilon}{4 \pi} \int_{S^{2}} \psi\left(\mathbf{x}, \boldsymbol{\Omega}^{\prime}\right) d \mathbf{\Omega}^{\prime}+\varepsilon \\
\mathbf{x} \in(0,1)^{2}, \boldsymbol{\Omega} \in D
\end{gathered}
$$

$$
\psi(\mathbf{x}, \mathbf{\Omega})
$$

$$
\begin{gathered}
=0 \quad \text { if } \quad \mathbf{x} \in \partial(0,1)^{2} \\
\text { and } \mathbf{n}(\mathbf{x}) \cdot \mathbf{\Omega}<0 .
\end{gathered}
$$
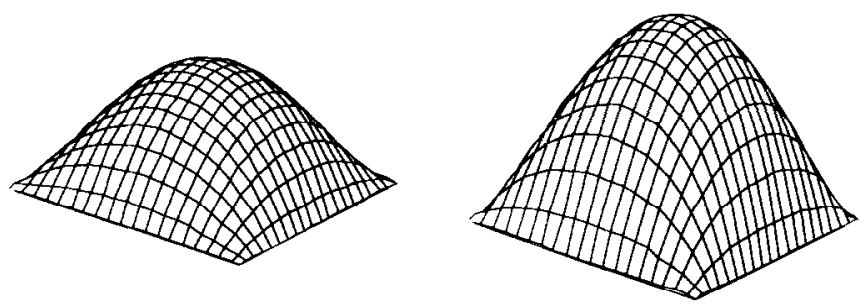

FIG. 2. $\varepsilon=10^{-4}, \operatorname{LD}$ (left) and $\operatorname{MLD}(\theta=1, \gamma=0)$ (right). 

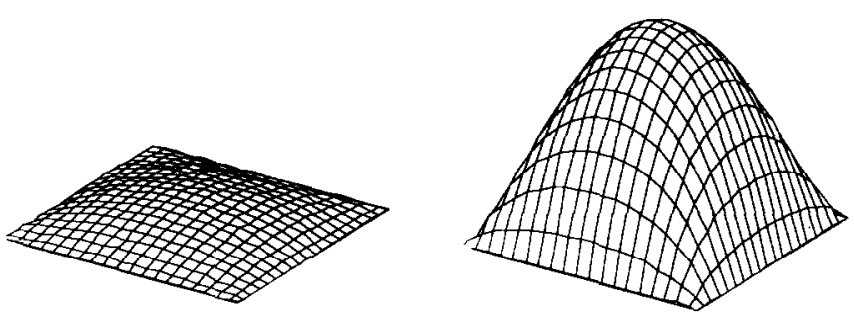

FIG. 3. $\varepsilon=10^{-5}, \operatorname{LD}$ (left) and $\operatorname{MLD}(\theta=1, \gamma=0)$ (right).

Here $\mathbf{n}(\mathbf{x})$ denotes the exterior unit normal vector on $\partial(0,1)^{2}$. We use the mesh

$$
\begin{aligned}
& x_{i+1 / 2}=\frac{i}{20}, \\
& y_{j+1 / 2}=\frac{j}{20}, \quad 0 \leqslant i, j \leqslant 20,
\end{aligned}
$$

and the $S_{2}$ quadrature scheme [2]. Figures 1-3 show results obtained using the LD scheme and using the MLD scheme with $\theta=1$ and $\gamma=0$, for three different values of $\varepsilon$. The plots indicate that the solutions obtained with the LD scheme tend to zero as $\varepsilon \rightarrow 0$, whereas the solutions obtained with the MLD scheme have a nonzero limit. Figure 4 shows the exact solution of the continuous diffusion problem

$$
\begin{aligned}
-\frac{1}{3} \Delta \Psi^{(0)}(\mathbf{x})+\Psi^{(0)}(\mathbf{x}) & =1, & & \mathbf{x} \in(0,1)^{2} \\
\Psi^{(0)}(\mathbf{x}) & =0, & & \mathbf{x} \in \partial(0,1)^{2}
\end{aligned}
$$

confirming that the diffusion limit of the MLD scheme is close to the solution of Eqs. (124) and (125).

\section{DISCUSSION AND OPEN QUESTIONS}

We believe that asymptotic studies of the kind presented in this paper are potentially useful for two reasons. First, a scheme that does not have the correct diffusion limit is likely to be inaccurate for many applications, in particular for elcctron and photon transport problems.

Second, an important problem is the efficient numerical solution of the very large systems of linear equations arising

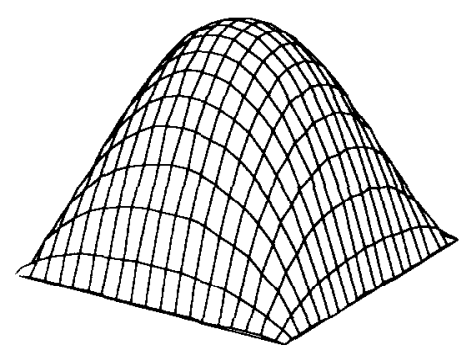

FIG. 4. Solution of diffusion problem. from discretizing two- or three-dimensional transport equations using discontinuous finite element methods (such as the one discussed in this paper), particularly for the case of large total collision cross sections and small absorption cross sections. It should be noted that the numerical linear algebra problem is more severe here than in the context of, for instance, standard two- or three-dimensional elliptic boundary value problems. The reason is that the number of independent variables is four in two space dimensions and five in three space dimensions. Our original motivation for the work described here was the hope that the asymptotic diffusion limit of the discrete scheme could be used, in a multi-level algorithm, to accelerate the source iteration method for the discretized transport problem, without obtaining a deterioration in convergence speed as the total collision cross section becomes large and the absorption cross section becomes small. We note that multi-level, or rather two-level methods using diffusion approximations to accelerate the source iteration method are well known. They are called diffusion synthetic acceleration (DSA) methods; see, for example, $[13,14]$ for an introduction to such methods. Subsequent to the completion of the work described in the present paper, Wareing, Larsen, and Adams [15] have shown that a modified bilinear discontinuous finite element discretization on rectangular meshes possesses a discrete diffusion limit consistent with the continuous one, and have developed a DSA method based on this result. We have only considered periodic problems in our analysis. A similar analysis is possibie for certain problems with boundaries. For example, in the case of vacuum boundary conditions, all solutions of the unmodified LD scheme tend to zero as $\varepsilon \rightarrow 0$. This was demonstrated numerically in Section 6 and can also be seen by applying the analysis of Section 4. However, the behavior of the modified scheme in the presence of nonperiodic boundaries needs further clarification. If, for instance, nonzero values are prescribed for the incoming fluxes on the boundary, the constant $A^{(0)}$ of Section 5 may be nonzero. Preliminary numerical results suggest that the scheme can produce unphysical oscillations in such cases. We also intend to perform similar studies for the linear discontinuous method on triangles and the bilinear discontinuous method on rectangles. We conjecture that both schemes have the correct behavior in the diffusion limit, without any modification. Nevertheless, modifications such as the ones discussed in this paper may be useful to improve the accuracy of the schemes for large $\sigma_{T}$ and small $\sigma_{A}$.

\section{ACKNOWLEDGMENTS}

Work by the first author (C. B.) was supported in part by NSF Grants DMS-8801991 and DMS-9003965, and by a Rackham Research Grant at the University of Michigan. A portion of the work of this author was carried out whilc he was a Visiting Scientist at the IBM Thomas J. Watson Research Center. Work by the second author (E. W. L.) was supported 
in part by NSF Grant EET-8721680, and by Los Alamos National Laboratory. Work by the third author (M. L. A.) was performed under the auspices of the U.S. Department of Energy at the Lawrence Livermore National Laboratory under Contract W-7405-ENG-48.

\section{REFERENCES}

1. K. M. Case and P. F. Zweifel, Linear Transport Theory (Addison-Wesley, Reading. MA, 1967).

2. E. E. Lewis and W. F. Miller, Jr., Computational Methods of Neutron Transport (Wiley-Interscience, New York, 1984).

3. E. W. Larsen and J. B. Keller, J. Math. Phys. 15, 75 (1974).

4. G. J. Habetler and B. J. Matkowsky, J. Math. Phys. 16, 846 (1975).

5. E. W. Larsen, J. E. Morel, and W. F. Miller, Jr., J. Comput. Phys. 69, 283 (1987).

6. E. W. Larsen and J. E. Morel, J. Comput. Phys. 83, 212 (1989).

7. T. R. Hill, "ONETRAN: A Discrete Ordinates Finite Element Code for the Solution of the One Dimensional Multigroup Transport Equation," Los Alamos Scientific Laboratory report LA-5990-MS, June, 1975 (unpublished).
8. M. Mordant, Ann. Nucl. Energy 8, 657 (1981).

9. M. Mordant, "New Phase Space Finite Element Transport Solutions," Proc. ANS Topical Meeting, Advances in Reactor Computations, Salt Lake City, March 28-31, 1983, Vol. 2, 783, 1983.

10. G. Samba, Nucl. Sci. Eng. 92, 197 (1986).

11. T. J. Seed, “TRIDENT-CTR User's Manual," Los Alamos Scientific Laboratory report LA-7835-M, revised, November, 1979 (unpublished).

12. P. Lesaint and-P. A. Raviart, "On a Finite Element Method for Solving the Neutron Transport Equation," in Mathematical Aspects of Finite Elements in Partial Differential Equations, edited by C. de Boor (Academic Press, New York, 1974).

13. R. E. Alcouffe, Nucl. Sci. Eng. 64, 344 (1977).

14. E. W. Larsen, Transport Th. Statist. Phys. 13, 107 (1984).

15. T. A. Wareing, E. W. Larsen, and M. L. Adams, "Diffusion Accelerated Discontinuous Finite Element Schemes for the $S_{N}$ Equations in Slab and $X, Y$ Geometries," Proc. ANS Topical Meeting, Advances in Mathematics, Computations, and Rcactor Physics, Pittsburgh, April 29-May 2, 1991, Vol. 3, Sec. 11.1, 2-1-2-12, 1991. 